\title{
PRODUCTS OF SHIFTED PRIMES SIMULTANEOUSLY TAKING PERFECT POWER VALUES
}

\author{
TRISTAN FREIBERG
}

\author{
(Received 2 March 2011; accepted 1 February 2012) \\ Communicated by I. E. Shparlinski \\ Dedicated to the memory of Alf van der Poorten
}

\begin{abstract}
Let $r$ be an integer greater than 1, and let $A$ be a finite, nonempty set of nonzero integers. We obtain a lower bound for the number of positive squarefree integers $n$, up to $x$, for which the products $\prod_{p \mid n}(p+a)$ (over primes $p$ ) are perfect $r$ th powers for all the integers $a$ in $A$. Also, in the cases where $A=\{-1\}$ and $A=\{+1\}$, we will obtain a lower bound for the number of such $n$ with exactly $r$ distinct prime factors.
\end{abstract}

2010 Mathematics subject classification: primary 11N25; secondary 11A25.

Keywords and phrases: shifted primes, Euler's totient function, perfect powers.

\section{Introduction}

If we pick a large integer close to $x$ at random, the probability that it is a perfect $r$ th power is around $x^{1 / r} / x$. We might expect the shifted primes $p+a$ to behave more or less like random integers in terms of their multiplicative properties. Thus, if we take a large squarefree integer $n$ close to $x$, we might naively expect that $\sigma(n)=\prod_{p \mid n}(p+1) \approx n$ is an $r$ th power with probability close to $x^{1 / r} / x$. However, as we will see, the probability is much higher than this, indeed more than $x^{0.7038} / x$, for any given $r$. We will even show that the likelihood of $\phi(n)$ and $\sigma(n)$ simultaneously being (different) $r$ th powers is more than $x^{0.2499} / x$. (As usual, $\phi$ denotes Euler's totient function and $\sigma$ denotes the sum-of-divisors function.) It would seem that $r$ th powers are 'popular' values for products of shifted primes in general.

Counting those $n$ with exactly $r$ prime factors, we will show that the number of such $n$ up to $x$ for which $\phi(n)$ is a perfect $r$ th power is at least of the order of $x^{1 / r} /(\log x)^{r+2}$, and likewise for $\sigma(n)$. Thus the number of positive integers $n$ such that $n \leq x$ and $n=p q$, where $p$ and $q$ are distinct primes, and $(p-1)(q-1)$ is a square, is at least of

Supported by the Göran Gustafsson Foundation (KVA).

(C) 2012 Australian Mathematical Publishing Association Inc. 1446-7887/2012 \$16.00 
the order of $x^{1 / 2} /(\log x)^{4}$. This may be seen as an 'approximation' to the well-known conjecture that there are infinitely many primes $p$ for which $p-1$ is a square. It is easily seen that, for any given $r \geq 2$, there is at most one prime $p$ such that $p+1$ is a perfect $r$ th power, namely $3+1=2^{2}, 7+1=2^{3}$, and so on.

Notation The expressions $F=O(G), F \ll G$, and $G \gg F$ all mean that $|F| \leq c G$, where $c$ is a positive constant. Further, $F \asymp G$ means that $F \ll G \ll F$. Where the constant $c$ is not absolute but depends on one or more parameters, this dependence may be indicated, as in, for example, $F \asymp_{\epsilon} G$, where the implied constants depend on $\epsilon$. If $f(x)$ and $g(x)$ are functions and $g(x)$ is nonzero for all sufficiently large $x$, we write $f(x) \sim g(x)$ to mean that $\lim _{x \rightarrow \infty} f(x) / g(x)=1$, and $f(x)=o(g(x))$ to mean that $\lim _{x \rightarrow \infty} f(x) / g(x)=0$. Other notation will be introduced as needed.

Given an integer $r \geq 2$ and a finite, nonempty set $A$ of nonzero integers, let

$$
\mathcal{B}(x ; A, r)=\left\{n \in S \cap[1, x]: \prod_{p \mid n}(p+a) \in \mathbb{Z}^{r} \forall a \in A\right\},
$$

where $S$ denotes the set of squarefree integers and $\mathbb{Z}^{r}$ denotes the set of perfect $r$ th powers. Banks et al. [4] proved, among other results, that

$$
|\mathcal{B}(x ;\{-1\}, 2)| \geq x^{0.7039-o(1)} \quad \text { and } \quad|\mathcal{B}(x ;\{+1\}, 2)| \geq x^{0.7039-o(1)} \text {, }
$$

and that

$$
|\mathcal{B}(x ;\{-1,+1\}, 2)| \geq x^{1 / 4-o(1)} .
$$

The first theorem generalizes both of these results.

THEOREM 1.1. Fix an integer $r \geq 2$, and a finite, nonempty set A of nonzero integers. As $x$ tends to infinity,

$$
|\mathcal{B}(x ; A, r)| \geq x^{1 / 2|A|-o(1)} .
$$

Moreover, if $|A|=1$, then as $x$ tends to infinity,

$$
|\mathcal{B}(x ; A, r)| \geq x^{0.7039-o(1)} .
$$

In the cases where $A=\{-1\}$ or $A=\{+1\}, \mathcal{B}(x ; A, r)$ is the set of positive squarefree integers $n$ up to $x$ for which $\phi(n)$ or $\sigma(n)$ respectively is an $r$ th power. There is no condition on the number of prime factors of $n$, but the next theorem concerns the sets

$$
\begin{aligned}
& \mathcal{B}^{*}(x ;-1, r)=\mathcal{B}(x ;\{-1\}, r) \cap\{n: \omega(n)=r\}, \\
& \mathcal{B}^{*}(x ;+1, r)=\mathcal{B}(x ;\{+1\}, r) \cap\{n: \omega(n)=r\},
\end{aligned}
$$

where $\omega(n)$ is the number of distinct prime factors of $n$.

Theorem 1.2. Fix an integer $r \geq 2$. For all sufficiently large $x$,

$$
\left|\mathcal{B}^{*}(x ;-1, r)\right| \gg \frac{r x^{1 / r}}{(\log x)^{r+2}} \quad \text { and } \quad\left|\mathcal{B}^{*}(x ;+1, r)\right| \gg \frac{r x^{1 / r}}{(\log x)^{r+2}} .
$$

The implied constants are absolute. 
The proof of Theorem 1.1, in Section 3, is an extension of the proof by Banks et al. [4] of the aforementioned special cases of Theorem 1.1. It employs some of the ideas of Erdős [9, 10] upon which Alford et al. [1] based their proof that there are infinitely many Carmichael numbers. (A Carmichael number is a composite number $n$ for which $a^{n} \equiv a \bmod n$ for all integers $a$.) Theorem 1.2 can be proved along the same lines. Indeed, in [5] it is shown that for all sufficiently large $x$, the lower bound $\left|\mathcal{B}^{*}(x ;-1, r)\right| \geq 4 x^{1 / r} /\left(9 e(\log x)^{2 r}\right)$ holds for $2 \leq r \leq(\log x /(12 \log \log x))^{1 / 2}$. However, our proof of Theorem 1.2, in Section 4, introduces a new method, which, as we will explain, is an application of the ideas of Goldston et al. [11].

\section{Preliminaries}

Theorem 1.1 is a consequence of the first four results of this section, and we use the fifth in the proof of Theorem 1.2.

An integer $n$ is called $y$-smooth if $p \leq y$ for every prime $p$ dividing $n$. Given a polynomial $F(X) \in \mathbb{Z}[X]$ and numbers $x \geq y \geq 2$, let

$$
\pi_{F}(x, y)=\mid\{p \leq x: F(p) \text { is } y \text {-smooth }\} \mid .
$$

In the case where $F=X-1$, Erdős [9] proved that there exists a number $\eta \in(0,1)$ such that $\pi_{F}\left(x, x^{\eta}\right) \gg_{\eta} \pi(x)$ (where $\pi(x)$ is the number of primes up to $x$ ), for all large $x$ depending on the choice of $\eta$. Several authors have improved upon this, the next two results being the best so far obtained.

Theorem 2.1. Fix a nonzero integer a and let $F(X)=X+a$. There exists an absolute constant c such that

$$
\pi_{F}(x, y)>\frac{x}{(\log x)^{c}}
$$

for all sufficiently large $x$, provided that $y \geq x^{0.2961}$.

Proof. See [2, Theorem 1].

Theorem 2.2. Let $F \in \mathbb{Z}[X]$. Let $g$ be the largest of the degrees of the irreducible factors of $F$ in $\mathbb{Z}[X]$, and let $k$ be the number of distinct irreducible factors of $F$ in $\mathbb{Z}[X]$ of degree $g$. Suppose that $F(0) \neq 0$ if $g=k=1$, and let $\epsilon$ be any positive real number. Then

$$
\pi_{F}(x, y) \asymp \frac{x}{\log x}
$$

for all sufficiently large $x$, provided that $y \geq x^{g+\epsilon-1 / 2 k}$.

Proof. See [6, Theorem 1.2].

For a finite additive abelian group $G$, denote by $n(G)$ the length of the longest sequence of (not necessarily distinct) elements of $G$, no nonempty subsequence of which sums to 0 , the additive identity of $G$. For instance, if $G=(\mathbb{Z} / 2 \mathbb{Z})^{m}$, then $n(G) \leq m$, for any sequence of $m+1$ elements of $G$ contains a nonempty subsequence 
whose elements sum to $(0, \ldots, 0) \bmod 2$, as can be seen by considering that such a sequence contains $2^{m+1}-1>2^{m}=|G|$ nonempty subsequences. For any group $G$ of order $m$, then any sequence of $m$ elements contains a nonempty subsequence whose sum is 0 , hence $n(G) \leq m-1$. The next theorem, due to van Emde Boas and Kruyswijk [8], gives a nontrivial upper bound for $n(G)$.

THEOREM 2.3. If $G$ is a finite abelian group and $m$ is the maximal order of an element in $G$, then $n(G)<m(1+\log (|G| / m))$.

Proof. See [8]. A proof is also given in [1, Theorem 1.1].

The following proposition shows that under certain conditions there are many sequences in $G$ whose elements sum to 0 .

Proposition 2.4. Let $G$ be a finite abelian group and let $r$ and $k$ be integers such that $r>k>n=n(G)$. Then any subsequence of $r$ elements of $G$ contains at least $\left(\begin{array}{l}r \\ k\end{array}\right) /\left(\begin{array}{l}r \\ n\end{array}\right)$ distinct subsequences of length at most $k$ and at least $k-n$, whose sum is the identity.

Proof. See [1, Proposition 1.2].

We will use the well-known Siegel-Walfisz theorem in the proof of Theorem 1.2.

Theorem 2.5 (Siegel-Walfisz). For any positive number B, there is a constant $C_{B}$ that depends only on B, such that

$$
\sum_{\substack{p \leq N \\ p \equiv a \bmod k}} \log p=\frac{N}{\phi(k)}+O\left(N \exp \left(-C_{B}(\log N)^{1 / 2}\right)\right)
$$

whenever $k \leq(\log N)^{B}$ and a is coprime with $k$.

Proof. See [7, Ch. 22].

\section{Proof of Theorem 1.1}

The following proof hinges on Theorem 2.3 and Proposition 2.4, which are key ingredients in the celebrated proof of Alford et al. [1] that there are infinitely many Carmichael numbers. In fact it is shown in [1, Theorem 1] that $C(x)$, the number of Carmichael numbers up to $x$, satisfies $C(x) \geq x^{\beta-\epsilon}$ for any positive $\epsilon$ and all sufficiently large $x$ (depending on the choice of $\epsilon$ ), where

$$
\beta=\frac{5}{12}\left(1-\frac{1}{2 \sqrt{e}}\right)=0.29036 \ldots
$$

Using a variant of the construction in [1], Harman [12] proved that $\beta=0.3322408$ is admissible, and, by combining the ideas of [1, 4, 12], Banks [3] established the following result. 
Theorem 3.1 [3, Theorem 1]. For every fixed $C<1$, there is a number $x_{0}(C)$ such that for all $x \geq x_{0}(C)$ the inequality

$$
\mid\{n \leq x: n \text { is Carmichael and } \phi(n) \text { is an rth power }\} \mid \geq x^{\beta-\epsilon}
$$

holds, where $\beta=0.3322408$ and $\epsilon$ is arbitrarily small but positive, for all positive integers $r \leq \exp \left((\log \log x)^{C}\right)$.

(Harman [13] subsequently proved that $\beta=0.7039 \times 0.4736>1 / 3$ is admissible here.) The method of the proof may yield further interesting results.

Theorems 2.1 and 2.2 are also crucial, and it will be manifest that extending the admissible range for $y$ in those theorems will lead to better estimates for $|\mathcal{B}(x ; A, r)|$. Explicitly, if $F(X)=\prod_{a \in A}(X+a)$ and

$$
\pi_{F}\left(x, x^{\eta}\right) \asymp_{F, \eta} \frac{x}{\log x},
$$

then the following proof yields $|\mathcal{B}(x ; A, r)| \geq x^{1-\eta-o(1)}$.

Proof of Theorem 1.1. Fix an integer $r \geq 2$ and a set $A=\left\{a_{1}, \ldots, a_{s}\right\}$ of nonzero integers. Let $x$ be a large number, and let

$$
y=\frac{\log x}{\log \log x} .
$$

Let $t=\pi(y)$, and let $G=(\mathbb{Z} / r \mathbb{Z})^{s t}$, so that by Theorem 2.3,

$$
n(G)<r(1+\log |G| / r)=r(1+(s t-1) \log r) .
$$

Fix any number $\epsilon \in(0,1 / 3 s)$, and let

$$
u= \begin{cases}0.2961^{-1} & \text { if } s=1 \\ \left(1+\epsilon-\frac{1}{2 s}\right)^{-1} & \text { if } s \geq 2\end{cases}
$$

Let

$$
F(X)=\left(X+a_{1}\right)\left(X+a_{2}\right) \cdots\left(X+a_{s}\right)
$$

and let

$$
\begin{aligned}
S_{F}\left(y^{u}, y\right) & =\left\{p \leq y^{u}: F(p) \text { is } y \text {-smooth }\right\} \\
& =\left\{p \leq y^{u}: p+a_{1}, \ldots, p+a_{s} \text { are } y \text {-smooth }\right\}
\end{aligned}
$$

We may suppose that $x$, and hence $y$, is large enough so that, by Theorems 2.1 and 2.2,

$$
\left|S_{F}\left(y^{u}, y\right)\right|=\pi_{F}\left(y^{u}, y\right) \gg \frac{y^{u}}{\left(\log y^{u}\right)^{c}}
$$


for some constant $c$. (We may suppose that $c=1$ if $s \geq 2$.) Finally, let

$$
k=\left[\frac{\log x}{\log y^{u}}\right],
$$

where $[\alpha]$ denotes the integer part of a real number $\alpha$.

By (3.1), (3.3) and (3.4),

$$
\frac{\pi_{F}\left(y^{u}, y\right)}{k} \gg \frac{(\log x)^{u-1}}{(\log \log x)^{u-1+c}},
$$

and by (3.1), (3.2) and (3.4),

$$
\frac{k}{n(G)} \gg_{r, s} \frac{\log x / \log y^{u}}{t} \gg \log \log x,
$$

because $t=\pi(y) \sim y / \log y$ as $y$ tends to infinity, by the prime number theorem. Hence, since $u>1$, we may assume $x$ is large enough that

$$
n(G)<k<\pi_{F}\left(y^{u}, y\right) .
$$

For primes $p \in S_{F}\left(y^{u}, y\right)$ and integers $a \in A$, we may write

$$
p+a=2^{\beta_{1}^{(a)}} 3^{\beta_{2}^{(a)}} \cdots p_{t}^{\beta_{t}^{(a)}},
$$

where $\beta_{i}^{(a)}$ are nonnegative integers when $1 \leq i \leq t$. We define

$$
\mathbf{v}_{p}=\left(\beta_{1}^{\left(a_{1}\right)}, \ldots, \beta_{t}^{\left(a_{1}\right)}, \beta_{1}^{\left(a_{2}\right)}, \ldots, \beta_{t}^{\left(a_{2}\right)}, \ldots, \beta_{1}^{\left(a_{s}\right)}, \ldots, \beta_{t}^{\left(a_{s}\right)}\right)
$$

as the 'exponent vector' for $p$. For a subset $R$ of $S_{F}\left(y^{u}, y\right), \prod_{p \in R}(p+a)$ is an $r$ th power for every $a \in A$ if and only if

$$
\sum_{p \in R} \mathbf{v}_{p} \equiv \mathbf{0} \bmod r
$$

where $\mathbf{0} \bmod r$ is the zero element of $G$. If, moreover, $R$ is of size at most $k$, then by (3.4),

$$
\prod_{p \in R} p \leq y^{u k} \leq x .
$$

Thus

$$
|\mathcal{B}(x ; A, r)| \geq \mid\left\{R \subseteq S_{F}\left(y^{u}, y\right):|R| \leq k \text { and } \sum_{p \in R} \mathbf{v}_{p} \equiv \mathbf{0} \bmod r\right\} \mid,
$$

as distinct subsets $R \subseteq S_{F}\left(y^{u}, y\right)$ give rise to distinct integers $n$, by the uniqueness of factorization.

Because of (3.6), we may deduce from Proposition 2.4 that the right-hand side of (3.7) is at least

$$
\left(\begin{array}{c}
\pi_{F}\left(y^{u}, y\right) \\
k
\end{array}\right) /\left(\begin{array}{c}
\pi_{F}\left(y^{u}, y\right) \\
n(G)
\end{array}\right) \geq\left(\frac{\pi_{F}\left(y^{u}, y\right)}{k}\right)^{k} \pi_{F}\left(y^{u}, y\right)^{-n(G)}=x^{f(x)},
$$


where

$$
f(x)=(k-n(G)) \frac{\log \pi_{F}\left(y^{u}, y\right)}{\log x}-\frac{k \log k}{\log x} .
$$

Letting $x$ tend to infinity and using (3.1), (3.3)-(3.5), we can now see that $f(x)=$ $1-1 / u-o(1)$. Therefore, as $x$ tends to infinity,

$$
|\mathcal{B}(x ; A, r)| \geq x^{1-1 / u-o(1)},
$$

and Theorem 1.1 follows by our choice of $u$, and letting $\epsilon$ tend to 0 if $s \geq 2$.

\section{Proof of Theorem 1.2}

We use a different approach to prove Theorem 1.2. The proof is 'inspired' by the breakthrough results of Goldston et al. [11] on short intervals containing primes. Basically, their proof begins with the observation that if $W(n)$ is a nonnegative weight and

$$
\sum_{N<n \leq 2 N}\left(\sum_{1 \leq h \leq H} \vartheta(n+h)-\log (2 N+H)\right) W(n)
$$

is positive, then for some $n \in(N, 2 N]$, the interval $(n, n+H]$ contains at least two primes. Here and later,

$$
\vartheta(n)= \begin{cases}\log n & \text { if } n \text { is prime } \\ 0 & \text { otherwise }\end{cases}
$$

Goldston et al. were able to obtain a nonnegative weight $W(n)$ for which the sum (4.1), with $H=\epsilon \log N$, is positive for all sufficiently large $N$. In our problem, we will be led to consider

$$
\sum_{1 \leq n \leq N}\left(\sum_{1 \leq a \leq H} \vartheta\left(a^{r} n+1\right)-(r-1) \log \left(H^{r} N+1\right)\right)
$$

(see (4.3)). A lower bound for this expression corresponds to a lower bound for the number of positive integers $n \leq N$ for which $\left\{a^{r} n+1: a \leq H\right\}$ contains at least $r$ primes. As we do not require $H$ to be 'short' compared to $N$, we may take $H=r \log N$; then the weight $W(n)=1$ works, and the problem is much easier.

Proof of Theorem 1.2. Throughout the proof, $r$ is a fixed integer greater than 1 , and $n, a, a_{1}, a_{2}, \ldots$ are positive integers. Observe that if, for some $n$, the numbers $\ell_{i}$, given by

$$
\ell_{i}=a_{i}^{r} n+1,
$$

are distinct primes (where $i=1, \ldots, r$ ), then

$$
\phi\left(\ell_{1} \cdots \ell_{r}\right)=\left(a_{1} \cdots a_{r} n\right)^{r} .
$$

If the primes $\ell_{i}$ are of the form $a_{i}^{r} n-1$ then $\sigma\left(\ell_{1} \cdots \ell_{r}\right)=\left(a_{1} \cdots a_{r} n\right)^{r}$. We will prove that $(1.1)$ holds for $|\mathcal{B}(x ;-1, r)|$, provided that $x$ is sufficiently large, and the same 
proof applies to $|\mathcal{B}(x ;+1, r)|$ if we consider primes of the form $a_{i}^{r} n-1$ rather than $a_{i}^{r} n+1$.

Let $N$ be a parameter tending monotonically to infinity and set $H=r \log N$. Let $\mathcal{A}(N)$ be the set of positive integers $n \leq N$ for which

$$
C_{n}:=\left\{a^{r} n+1: a \leq H\right\} \cap \mathcal{P}
$$

(where $\mathcal{P}$ is the set of all primes) contains at least $r$ primes. We will show that

$$
|\mathcal{A}(N)| \gg \frac{N}{\log N},
$$

but first we will describe how this implies a lower bound for $|\mathcal{B}(x ;-1, r)|$.

Every $n \in \mathcal{A}(N)$ gives rise, via $C_{n}$, to some $\ell_{1} \cdots \ell_{r} \in \mathcal{B}\left(\left(H^{r} N+1\right)^{r} ;-1, r\right)$, though different $n$ may give rise to the same $r$-tuple of primes. On the other hand, given $n \in \mathcal{A}(N)$ and a prime $p=a^{r} n+1 \in C_{n}$, each $m \in \mathcal{A}(N)$ for which $p \in C_{m}$ corresponds to a solution to $a^{r} n=b^{r} m, b \leq H$. Therefore there can be at most $H$ different integers $n \in \mathcal{A}(N)$ giving rise to the same element of $\mathcal{B}\left(\left(H^{r} N+1\right)^{r} ;-1, r\right)$. Consequently,

$$
\left|\mathcal{B}\left(\left(H^{r} N+1\right)^{r} ;-1, r\right)\right| \geq \frac{|\mathcal{A}(N)|}{H} \gg \frac{N}{r(\log N)^{2}}
$$

by (4.2), and (1.1) follows.

We will now establish (4.2). We will show that for all large $N$,

$$
S(N)=\sum_{1 \leq n \leq N}\left(\sum_{1 \leq a \leq H} \vartheta\left(a^{r} n+1\right)-(r-1) \log \left(H^{r} N+1\right)\right) \gg r N \log N .
$$

Consequently $\mathcal{A}(N)$ is nonempty for large $N$. Indeed, if (4.3) holds then

$$
\begin{aligned}
r N \log N \ll S(N) & \leq \sum_{n \in \mathcal{A}(N)}\left(\sum_{1 \leq a \leq H} \vartheta\left(a^{r} n+1\right)-(r-1) \log \left(H^{r} N+1\right)\right) \\
& \leq|\mathcal{A}(N)| H \log \left(H^{r} N+1\right),
\end{aligned}
$$

and (4.2) follows because $\log \left(H^{r} N+1\right) \sim \log N$.

For the evaluation of $S(N)$, first note that

$$
\sum_{1 \leq n \leq N} \sum_{1 \leq a \leq H} \vartheta\left(a^{r} n+1\right)=\sum_{1 \leq a \leq H} \sum_{\substack{p \leq a^{r} N+1 \\ p \equiv 1 \bmod a^{r}}} \log p
$$

Since $a^{r} \ll_{r}(\log N)^{r}$ for $a \leq H$, we may apply Theorem 2.5 to the last sum. We have

$$
\sum_{\substack{p \leq a^{r} N+1 \\ p \equiv 1 \bmod a^{r}}} \log p=\frac{a^{r} N}{\phi\left(a^{r}\right)}+O\left(\frac{a^{r} N}{\phi\left(a^{r}\right)(\log N)^{2}}\right) \sim \frac{a}{\phi(a)} N .
$$


Therefore, from the well-known estimate

$$
\sum_{1 \leq a \leq H} \frac{a}{\phi(a)} \sim c H \quad \text { where } c=\prod_{p}\left(1+\frac{1}{p(p-1)}\right)=1.943596 \ldots,
$$

we deduce that

$$
\sum_{1 \leq n \leq N} \sum_{1 \leq a \leq H} \vartheta\left(a^{r} n+1\right) \sim N \sum_{1 \leq a \leq H} \frac{a}{\phi(a)} \sim c N H
$$

Also,

$$
\sum_{1 \leq n \leq N}(r-1) \log \left(H^{r} N+1\right) \sim N(r-1) \log N,
$$

so combining all of this yields

$$
S(N) \sim N(c H-(r-1) \log N) \gg r N \log N,
$$

and (4.3) follows.

\section{Acknowledgements}

I would like to thank Andrew Granville for introducing me to the problems considered in this paper, and for his help in its preparation. I thank William Banks for bringing [5] to my attention, and Christian Elsholtz for some helpful comments. Finally, I am grateful to the anonymous referee for providing comments and corrections.

\section{References}

[1] W. R. Alford, A. Granville and C. Pomerance, 'There are infinitely many Carmichael numbers', Ann. of Math. (2) 139 (1994), 703-722.

[2] R. C. Baker and G. Harman, 'Shifted primes without large prime factors', Acta Arith. 83 (1998), 331-361.

[3] W. D. Banks, 'Carmichael numbers with a square totient', Canad. Math. Bull. 52 (2009), 3-8.

[4] W. D. Banks, J. B. Friedlander, C. Pomerance and I. E. Shparlinski, 'Multiplicative structure of values of the Euler function', in: High Primes and Misdemeanours: Lectures in Honour of the 60th Birthday of Hugh Cowie Williams, Fields Institute Communications, vol. 41 (eds. A. van der Poorten and A. Stein) (American Mathematical Society, Providence, RI, 2004), pp. 29-47.

[5] W. D. Banks and F. Luca, 'Power totients with almost primes', Integers 11 (2011), 307-313.

[6] C. Dartyge, G. Martin and G. Tenenbaum, 'Polynomial values free of large prime factors', Period. Math. Hungar. 43 (2001), 111-119.

[7] H. Davenport, Multiplicative Number Theory, 3rd edn (Springer, New York, 2000), revised and with a preface by H. L. Montgomery.

[8] P. van Emde Boas and D. Kruyswijk, A Combinatorial Problem on Finite Abelian Groups III, Afd. zuivere Wisk., 1969-008 (Math. Centrum, Amsterdam, 1969).

[9] P. Erdős, 'On the normal number of prime factors of $p-1$ and some other related problems concerning Euler's $\phi$-function', Q. J. Math. (Oxford Ser.) 6 (1935), 205-213.

[10] P. Erdős, 'On pseudoprimes and Carmichael numbers', Publ. Math. Debrecen 4 (1956), 201-206. 
[11] D. A. Goldston, J. Pintz and C. Y. Yıldırım, 'Primes in tuples I', Ann. of Math. (2) 170 (2009), 819-862.

[12] G. Harman, 'On the number of Carmichael numbers up to $x$ ', Bull. Lond. Math. Soc. 37 (2005), 641-650.

[13] G. Harman, 'Watt's mean value theorem and Carmichael numbers', Int. J. Number Theory 4 (2008), 241-248.

TRISTAN FREIBERG, Department of Mathematics, KTH Royal Institute of Technology, SE-100 44 Stockholm, Sweden e-mail: tristanf@kth.se 